(C) 2019 IEEE. Personal use of this material is permitted. Permission from IEEE must be obtained for all other uses, in any current or future media, including reprinting/republishing this material for advertising or promotional purposes, creating new collective works, for resale or redistribution to servers or lists, or reuse of any copyrighted component of this work in other works. 


\title{
InP PIC's Scalability for Datacenter Applications
}

\author{
B. Docter, K. Solis-Trapala, A. Albores-Mejia \\ EFFECT Photonics B.V., Torenallee 20, 5617 BC Eindhoven, The Netherlands \\ b.docter@effectphotonics.nl
}

\begin{abstract}
InP PIC fabrication platforms have seen an accelerated development over the recent years. Monolithic integration allows novel concepts in packaging and testing technology. Altogether they enable a ready to scale cost-effective InP PIC ecosystem.
\end{abstract}

\section{Summary}

InP PIC technology platform is the most versatile monolithic integration platform for photonic circuits. The ability to integrate both high-performance (tunable-) lasers with high-speed low loss modulators, as well as passive functions and detectors, makes the technology very appealing for a wide range of modern telecommunication applications. The technology is currently being used in integrated tunable laser/modulator circuits, both for common $10 \mathrm{~Gb} / \mathrm{s}$ direct detect products by e.g. Finisar, Sumitomo, and Lumentum, as well as for $100+\mathrm{Gb} / \mathrm{s}$ coherent transceivers products by e.g. Oclaro, Neophotonics, and Infinera.

Most commercial products that employ InP PICs are produced in vertically integrated fabrication facilities in which the production line is dedicated to a small set of products, e.g. [1]. This makes the initial investment and therefore the barrier to entry for new products very high. In the last decade, multiple research institutes have pioneered a novel foundry approach to InP PIC manufacturing. Several Multi-Project Wafer (MPW) services have emerged [2,3], allowing new device concepts to be developed at a low cost using standardized building blocks.

In this presentation, we will analyze the technology readiness of monolithic InP PIC technology. Particularly, we will look at the ability to address the volume and cost requirements as set out by the current datacenter market segment. We will discuss the opportunities and remaining challenges that monolithic InP PIC's bring to the packaging and testing technology. Finally, we will look at the applications within the datacenter market that will profit, short and long term, from this scalable cost-effective InP PIC ecosystem.

\section{References}

[1] G.E. Hoefler, et al., “Tb/s-Class InP System-On-Chip Photonic ICs,” European Conference on Integrated Optics (ECIO), Th.3.1-2, (2018).

[2] JePPiX, “Technology Roadmap" in JePPIX ROADMAP 2018: THE ROAD TO A MULTI-Billion EURO MARKET IN INTEGRATED PHOTONICS Chap. 5., (2018).

[3] F. M. Soares et al., "High-Performance InP PIC Technology Development based on a Generic Photonic Integration Foundry," 2018 Optical Fiber Communications Conference and Exposition (OFC), San Diego, CA, 2018, pp. 1-3. 